\title{
Producción y rentabilidad de cuyes alimentados con arbustivas forrajeras tropicales en zona rural de Quevedo, Ecuador
}

\section{Profitability and production of guinea pigs fed with forage shrubs tropical in rural area of Quevedo, Ecuador}

\author{
Meza Bone Carlos Javier ${ }^{*}$, Cabrera Verdezoto Rodrigo Paúl ${ }^{1,2}$, Morán Morán Jessica Jessenia ${ }^{3}$, Cabrera Verdesoto Cesar \\ Alberto $^{3}$, Mieles Cedeño Eva Moncerrate ${ }^{2}$, Meza Bone Gary Alex ${ }^{1,2}$ \\ ${ }^{1}$ Universidad Técnica Estatal de Quevedo, Facultad de Ciencias Pecuarias. Carrera de Ingeniería Agropecuaria, Campus Finca Experimental \\ “La María”. CP. 121250 Km. 7 1⁄2 vía El Empalme, cantón Mocache.Los Ríos.Ecuador.cmeza@uteq.edu.ec, gmeza@uteq.edu.ec \\ ${ }^{2}$ Instituto Tecnológico Superior Ciudad de Valencia. Campus Extensión de la Universidad de Babahoyo El Pital 1, Km. 3 1/2 vía a Valencia, \\ cantón Quevedo. Los Ríos. Ecuador. \\ ${ }^{3}$ Universidad Estatal del Sur de Manabi, Facultad de Ciencias Naturales y de la Agricultura. Carrera de Ingeniería Agropecuaria y Forestal. \\ Campus "Los Ángeles". Km. 1 1/2 vía a Noboa, cantón Jipijapa. Manabí. Ecuador.
}

Rec.: 30.10.2018. Acept.: 13.12.2018 Publicado el 30 de diciembre de 2018

\begin{abstract}
Resumen
$\mathrm{E}$ 1 objetivo del estudio fue determinar el comportamiento productivo y rentabilidad de cuyes (Cavia porcellus) bajo el efecto de consumo de forrajeras tropicales (Morus alba, Erythrina poeppigiana y Tithonia diversifolia). Los tratamientos fueron $\mathrm{T} 1=$ Balanceado ad libitum; $\mathrm{T} 2=$ Balanceado $15 \mathrm{~g}+$ M. alba ad libitum; T3= Balanceado $15 \mathrm{~g}+$ E. poeppigiana $\mathrm{ad}$ libitum y $\mathrm{T} 4=$ Balanceado $15 \mathrm{~g}+\mathrm{T}$. diversifolia ad libitum. Se aplicó un diseño completo al azar con seis repeticiones, la unidad experimental estuvo conformada por dos gazapos, se utilizaron 48 cuyes machos de 30 días de edad y un peso promedio de 363,54 $\mathrm{g}$, para establecer la diferencia entre medias se aplicó la prueba de Tukey $(\mathrm{P} \leq 0,05)$. Se evaluó el consumo de balanceado $(\mathrm{CB}) \mathrm{g}$, consumo de forraje (CF) g, consumo de alimento total (CAT) g, ganancia de peso (GP) g, índice de conversión alimenticia (ICA) y rendimiento a la canal $(\mathrm{RC}) \%$. La rentabilidad se determinó a través de la relación beneficio-costo. El mayor CF; GP; RC y la rentabilidad más eficiente tuvieron los tratamientos T2 y T3 (37,83 y 36,85 g MS animal $\left.{ }^{-1} \mathrm{~d}^{-1}\right)$. T1 y T2 (10,62 y 9,53 g animal $\left.^{-1} \mathrm{~d}^{-1}\right) \mathrm{T} 2$, T3 y T1 $(71,93 ; 66,14$ y 64,28\%) y T2 (64,48\%), respectivamente. El mayor ingreso de venta por carne producida y el mayor beneficio/costo tuvo el tratamiento T2 ( $\$ 5,93$ cuy1 y 1,64 cuy-1), representando el punto de equilibrio de $\$ 3,44$ animal $^{-1}$ lo cual demuestra que es una buena alternativa para la crianza de cuyes.
\end{abstract}

Palabras clave: Parámetros, comportamiento productivo, balanceado, alimentación.
$\mathrm{T}$ The objective of the study was to determine the productive behavior and profitability of guinea pigs (Cavia porcellus) under the effect of consumption of tropical forages (Morus alba, Erythrina poeppigiana and Tithonia diversifolia). The treatments were $\mathrm{T} 1=$ Balanced ad libitum; T2 = Balanced $15 \mathrm{~g}+$ M. alba ad libitum; T3 = Balanced $15 \mathrm{~g}+$ E. poeppigiana ad libitum and $\mathrm{T} 4=$ Balanced $15 \mathrm{~g}+T$. diversifolia ad libitum. A complete randomized design was applied with six repetitions, the experimental unit was made of two guinea pigs, 48 male guinea pigs of 30 days of age and average weight of 363.54 $\mathrm{g}$ were used. To establish the difference between means, the Tukey test $(\mathrm{P} \leq 0.05)$ was applied. Balanced consumption (CB) g, forage intake (CF) g, total feed consumption (CAT) g, weight gain (GP) g, feed conversion ratio (ICA) and carcass yield (RC) \% were evaluated. Profitability was determined through the benefit-cost relationship. The best CF; GP; RC and the most efficient profitability were recorded by treatments $\mathrm{T} 2$ and $\mathrm{T} 3$ (37,83 and 36,85 g MS animal $\left.^{-1} \mathrm{~d}^{-1}\right)$. T1 and T2 $\left(10,62\right.$ and 9,53 $\mathrm{g}$ animal $\left.^{-1} \mathrm{~d}^{-1}\right) \mathrm{T} 2$, T3 and T1 (71,93; 66,14 and 64,28\%) and T2 (64,48\%), respectively. The highest sales income for meat produced and the highest benefit/cost was recorded by treatment T2 $\left(\$ 5,93\right.$ cuy $^{-1}$ and 1,64 cuy $\left.^{-1}\right)$, representing the equilibrium point of $\$ 3,44$ animal $^{-1}$ it shows that is a good alternative for raising guinea pigs.

Keywords: Parameters, behaviour productive, balanced, food. 


\section{Introducción}

$\mathrm{E}$ cuy es un pequeño roedor originario de los Andes, utilizado como alimento en una extensa región comprendida por Chile, Argentina, Bolivia, Perú, Ecuador y Colombia. Su crianza es generalizada en el ámbito rural como un animal de carne para autoconsumo, constituyéndose en una excelente alternativa para diversificar la dieta. Considerado por la ONU y la FAO como una fuente de seguridad alimenticia de la población mundial de escasos recursos económicos (Sánchez et al., 2009). Así, la nutrición juega un rol importante en toda explotación pecuaria, el adecuado suministro de nutrientes conlleva a una mejor producción. El conocimiento de los requerimientos nutritivos de los cuyes nos permitirá poder elaborar raciones balanceadas que logren satisfacer las necesidades en cada una de las fases biológicas por las que atraviesa (gestación, lactancia, engorde) (Meza et al., 2014). El uso de recursos fibrosos en dietas para cuyes influye en el proceso digestivo, aprovechamiento de nutrientes y eficiencia biológica debido a cambios en la tasa de pasaje. Por esta razón, es necesario conocer el contenido de nutrientes y la valoración nutricional de esos recursos alimenticios, representando un interesante tema de estudio que puede contribuir a mejorar la formulación de dietas para cuyes.

Las plantas arbustivas forrajeras adaptadas al medio tropical y de fácil adquisición, son un recurso para la alimentación eficiente de cuyes en el Litoral ecuatoriano, en el manejo de la alimentación se fusionan los conocimientos científicos y prácticos, con la finalidad de hacer más productiva la especie, sin detrimento de la economía del productor (Sánchez et al., 2012). La tendencia actual de utilizar forrajes de origen arbustivo o arbóreo (M. alba, E. poeppigiana , $T$. diversifolia), es estimulado debido a los incrementos de los precios de los granos de cereales y oleaginosas, lo que además de incrementar los costos de producción animal, tiene el inconveniente que compiten con la alimentación humana. Por tal motivo, se busca engordar cuyes usando forrajes que posean gran valor nutricional que aseguren cubrir los requerimientos nutricionales. Por tanto, estos forrajes tienen innumerables ventajas, entre ellas, poseer un alto valor nutritivo que constituyen una solución en la alimentación de cuyes en el trópico (Cáceres et al., 1996). De allí que, es importante realizar un análisis económico de todos estos factores para calcular su rentabilidad, pues, en cada uno de ellos se manifestarían los efectos sobre los costos del engorde de cuyes y de los ingresos por los rendimientos de los mismos. Por tanto, el objetivo del presente experimento fue determinar el comportamiento productivo y la rentabilidad de cuyes alimentados con arbustivas forrajeras tropicales.

\section{Materiales y métodos}

T a investigación se ejecutó en la Quinta "La Fase" $\bigcup_{\text {propiedad del Ing. Alejandro Meza Chica, localizada en }}$ el km 8 de la vía Quevedo-Mocache, provincia de Los Ríos cuya ubicación geográfica de $1^{\circ} 6^{\prime} 23^{\prime \prime}$ de latitud sur y $79^{\circ} 29^{\prime}$ 12 " de longitud oeste y a una altura de $70 \mathrm{msnm}$.

Se utilizaron 48 cuyes machos de 30 días de edad, pesados en una balanza gramera electrónica tipo mesa, marca Electrolux KS05B de capacidad de $5 \mathrm{~kg}$ de precisión. posteriormente los animales fueron alojados en 24 jaulas de malla galvanizada de $0,50 \mathrm{~m}$ de largo, $0,40 \mathrm{~m}$ de ancho y $0,35 \mathrm{~m}$ de alto, con sus respectivos comederos de tipo tol y bebederos tipo chupón en un galpón de $30 \mathrm{~m}^{2}$, con una temperatura ambiental promedio de $24,70 \pm 3{ }^{\circ} \mathrm{C}$. La fase experimental duró 56 días. Estos tuvieron un periodo de adaptación de 4 días consumiendo forrajes tropicales en fresco. Los cuyes se distribuyeron de acuerdo al diseño completamente al azar. Cuatro tratamientos, seis réplicas y dos cuyes por unidad experimental. Para determinar la diferencia en media de tratamientos se aplicó la prueba de rangos múltiples de Tukey $(\mathrm{p}<0,05)$, utilizando el procedimiento de los modelos lineales generales (GLM) del SAS (2000).

Los tratamientos de prueba fueron $\mathrm{T} 1=$ testigo (balanceado ad libitum); T2= balanceado $15 \mathrm{~g}+$ Morus alba ad libitum; T3= balanceado $15 \mathrm{~g}+$ Erythrina poeppigiana ad libitum y $\mathrm{T} 5=$ balanceado $15 \mathrm{~g}+$ Tithonia diversifolia $\mathrm{ad}$ libitum.

Se evaluó el consumo de forraje $(\mathrm{CF})$, consumo de balanceado (CB), consumo de alimento total (CAT), ganancia de peso (GP), índice de conversión alimenticia (ICA), peso a la canal (PC) y rendimiento a la canal (RC). La rentabilidad fue determinada por la relación beneficio-costo. El alimento se suministró diariamente (dos veces al día) previamente pesado (g) a las (07:00 am y 16:00 pm) y se determinó el consumo a través de la diferencia entre lo ofrecido y lo rechazado. La ganancia de peso fue la resta entre el peso anterior y el peso actual. La conversión de alimento se determinó entre el consumo de alimento consumido y el peso vivo ganado por los cuyes durante el periodo de engorde. Mientras, el rendimiento a la canal se obtuvo dividiendo peso a la canal para la ganancia de peso. Para el análisis de la gestión financiera se utilizó el cálculo a partir de un presupuesto parcial, análisis de dominancia y análisis marginal para obtener los costos fijos y variables, beneficio neto, relación beneficio costo, rentabilidad y punto de equilibrio; para éste último indicador se tomó en consideración la máxima producción de carne.

\section{Resultados y discusión}

$\mathrm{E}^{\mathrm{l}}$ mayor consumo de alimento (Cuadro 1) lo registraron $(\mathrm{p}<0,01)$ los tratamientos testigo, M. alba y E. poeppigiana $\left(55,50 ; 51,62\right.$ y 50,64 g MS animal ${ }^{-1}$ día $\left.^{-1}\right)$. Estos resultados superan a los reportados por Apráez et al. (1997) al utilizar $70 \%$ de pasto king grass más $30 \%$ de concentrado, oteniendo consumos de alimento de 38,85 g MS animal $\mathrm{d}^{-1}$. Mientras, Apraez et al. (2001), al estudiar el pasto kikuyo orgánico 50\%, mineral 50\%, + $30 \mathrm{~g}$ de concentrado, reportaron valores de 
Cuadro 1. Comportamiento productivo en cuyes de engorde alimentados con balanceados y arbustivas forrajeras tropicales

\begin{tabular}{lccccc}
\hline & $\begin{array}{c}\text { Balanceado } \\
\text { ad libitum }\end{array}$ & $\begin{array}{c}\text { M. alba } \\
\text { ad libitum }\end{array}$ & $\begin{array}{c}\text { E. poeppigiana } \\
\text { ad libitum }\end{array}$ & $\begin{array}{c}\text { T. diversifolia } \\
\text { ad libitum }\end{array}$ & \multirow{2}{*}{ CV (\%) } \\
\cline { 1 - 5 } $\mathrm{N}$ & 12 & 12 & 12 & 12 & \\
\hline $\mathrm{CAB}\left(\mathrm{g} \mathrm{cuy}^{-1}\right.$ día $\left.^{-1}\right)$ & $55,50 \mathrm{a}$ & $12,71 \mathrm{~b}$ & $12,71 \mathrm{~b}$ & $12,71 \mathrm{~b}$ & 31,63 \\
$\mathrm{CAF}\left(\mathrm{g} \mathrm{cuy}^{-1}\right.$ día $\left.^{-1}\right)$ & ---- & $37,83 \mathrm{a}$ & $36,85 \mathrm{a}$ & $23,25 \mathrm{~b}$ & 14,90 \\
$\mathrm{CA}\left(\mathrm{g} \mathrm{cuy}^{-1}\right.$ día $\left.^{-1}\right)$ & $55,50 \mathrm{a}$ & $51,62 \mathrm{a}$ & $50,64 \mathrm{a}$ & $37,04 \mathrm{~b}$ & 17,25 \\
$\mathrm{GP}\left(\mathrm{g} \mathrm{cuy}^{-1}\right.$ día $\left.^{-1}\right)$ & $10,62 \mathrm{a}$ & $9,53 \mathrm{ab}$ & $8,33 \mathrm{~b}$ & $7,85 \mathrm{~b}$ & 11,49 \\
$\mathrm{ICA}$ & $5,38 \mathrm{a}$ & $5,50 \mathrm{a}$ & $6,18 \mathrm{a}$ & $5,00 \mathrm{a}$ & 14,46 \\
$\mathrm{RC}(\%)$ & $64,28 \mathrm{a}$ & $71,93 \mathrm{a}$ & $66,14 \mathrm{a}$ & $53,82 \mathrm{~b}$ & 8,60 \\
\hline CAB=Consumo de alimento & balanceado. CAF=Consumo de alimento de forraje. CA=Consumo de alimento.
\end{tabular}

$\mathrm{GP}=$ Ganancia de peso. $\mathrm{ICA}=$ =́ndice de conversión alimenticia. $\mathrm{RC}=$ Rendimiento a la canal.

23,19 g MS animal $\mathrm{d}^{-1}$. Camino y Hidalgo (2014) al evaluar dos genotipos de cuyes (C. porcellus) alimentados con concentrado reportaron consumos de alimento de 49,20 g MS animal ${ }^{-1} \mathrm{~d}^{-1}$.

Sin embargo, los consumos de alimento en la presente investigación son inferiores a los presentados por Apráez et al. (2013) al estudiar el comportamiento productivo de cuyes, bajo arreglos silvopastoriles en clima medio, utilizando Axonopus scoparius + concentrado comercial, quienes obtuvieron valores de 81,95 g MS animal ${ }^{-1} \mathrm{~d}^{-1}$. Asimismo, Sánchez et al. (2013) al estudiar la respuesta productiva y económica al uso de cuatro tipos de comederos para forraje en la crianza de cuyes al utilizar malla colgante en forma de $\mathrm{U}$ reporta valores de 79,30 $\mathrm{g} \mathrm{MS}_{\text {animal }}{ }^{-1} \mathrm{~d}^{-1}$.

El mayor consumo de alimento balanceado $(\mathrm{P} \leq 0,01)$ lo registró el tratamiento testigo $\left(55,50 \mathrm{~g} \mathrm{MS}\right.$ animal ${ }^{-1}$ día $\left.^{-1}\right)$, resultados que son superiores a los publicados por Ramos et al. (2013) en la valoración de la harina de nabo (Brassica campestris) en alimentación de cuyes, obteniendo valores de 31,80 g MS animal ${ }^{-1} \mathrm{~d}^{-1}$. Entretanto, Castillo et al. (2012) estudiaron el efecto de la suplementación con bloques minerales sobre la productividad de cuyes alimentados con forraje saboya y concentrado, obteniendo un consumo en concentrado de $31,35 \mathrm{~g} \mathrm{MS}_{\text {animal }}{ }^{-1} \mathrm{~d}^{-1}$.

Los mayores consumos de forraje $(\mathrm{P} \leq 0,01)$ lo registraron los tratamientos $M$. alba y E. poeppigiana $(37,83$ y $36,85 \mathrm{~g}$ MS animal $\left.{ }^{-1} \mathrm{~d}^{-1}\right)$. Sin embargo, estos resultados son inferiores a los reportados por Ramos et al. (2013) quienes evaluaron el comportamiento productivo de cuyes alimentados con pasto Lolium sp., y forraje de abutilón (Abutilon striatum), obteniendo valores de 63,05 $\mathrm{g} \mathrm{MS}$ animal $^{-1} \mathrm{~d}^{-1}$. Quintana et al. (2013) registraron valores de 40,33 $\mathrm{g} \mathrm{MS}$ animal $^{-1} \mathrm{~d}^{-1}$ cuando valoraron el efecto de dietas de alfalfa verde, harina de cebada y bloque mineral sobre la eficiencia productiva de cuyes. Lozada et al. (2013) trabajaron con dietas que incluyeron de cebada en grano y semilla de girasol en una alimentación a base de forraje obteniendo un consumo de forraje de $67,88 \mathrm{~g}$
MS animal ${ }^{-1} \mathrm{~d}^{-1}$. Jiménez et al. (2000) hallaron valores de 57,00 y 59,60 $\mathrm{g} \mathrm{MS}$ animal $^{-1} \mathrm{~d}^{-1}$ al determinar el momento óptimo económico de beneficio en cuyes alimentados con alfalfa vs una suplementación de afrechillo, ambos suministrado ad libitum. De igual manera Apráez et al. (2008) obtuvieron valores de 57,31 $\mathrm{g} \mathrm{MS}_{\text {animal }}{ }^{-1} \mathrm{~d}^{-1}$ en el consumo de $M$. alba. Igualmente Forte y Fernández (1994) y Savón et al. (2006) evaluaron el uso del follaje de $M$. alba en la alimentación de cuyes y registraron consumos de alimento para la Morus alba y Erythrina poeppigiana de 56,00 y 55,50 $\mathrm{g} \mathrm{MS}_{\text {animal }}{ }^{-1}$ $\mathrm{d}^{-1}$. Mientras, Alata et al. (2008) publicaron valores de 75,0 g MS animal ${ }^{-1} \mathrm{~d}^{-1}$ en cuyes alimentados con Pisonay (Erythrina falcata). Sin embargo, estos resultados superan a los reportados por Zeballos et al. (1994) quienes indican consumos de 23,5 y 40,3 $\mathrm{g} \mathrm{MS}$ animal $^{-1} \mathrm{~d}^{-1}$ en la valoración de la hoja de $M$. alba mediante pruebas de digestibilidad y crecimiento.

Las mejores ganancias de peso $(\mathrm{P} \leq 0,01)$ lo registraron los tratamientos testigo y $M$. alba (10,62 y 9,53 $\left.\mathrm{g}_{\text {animal }}{ }^{-1} \mathrm{~d}^{-1}\right)$. Resultados que son superiores a los obtenidos por Apráez et al. (1997) en la valoración de dos leguminosas nativas zarza (Mimosa albida) y pepa pega (Desmodium tortuosum) en la alimentación de cuyes, consiguieron pesos de 4,95 $\mathrm{g}$ animal ${ }^{-1} \mathrm{~d}^{-1}$. También, Apráez et al. (2001) publicaron ganancias de $2,83 \mathrm{~g}_{\text {animal }}^{-1} \mathrm{~d}^{-1}$ cuando utilizaron pasto kikuyo orgánico $50 \%$, mineral $50 \%+30$ g de concentrado. Mienstras tanto, Castillo et al. (2012) evaluaron el efecto de la suplementación con bloques minerales sobre la productividad de cuyes alimentados con forraje obteniendo una ganancia de peso 5,68 g animal $^{-1} \mathrm{~d}^{-1}$.

Los resultados obtenidos en la presente investigación, se asemejan a los publicados por Apráez et al. (2013) cuando estudiaron el comportamiento productivo de cuyes, bajo arreglos silvopastoriles en clima medio, utilizando A. scoparius ad libitum + concentrado comercial $15 \mathrm{~g}$, obteniendo ganancias de peso de $8,75 \mathrm{~g}_{\text {animal }}{ }^{-1} \mathrm{~d}^{-1}$. Ramos et al. (2013) también evaluaron el comportamiento 
productivo de cuyes alimentados con pasto Lolium sp., y forraje de abutilón (Abutilon striatum) ad libitum, consiguiendo 7,34 g animal ${ }^{-1} \mathrm{~d}^{-1}$. Quintana et al., (2013) estudiaron el efecto de dietas con alfalfa verde, harina de cebada ad libitum y bloque mineral sobre la eficiencia productiva de cuyes y obtuvieron ganancias de 7,88 g animal ${ }^{-1} \mathrm{~d}^{-1}$. Lozada et al. (2013) informaron el efecto de la inclusión de cebada grano y semilla de girasol en una dieta basada en forraje sobre el momento óptimo de beneficio de cuyes obteniendo una ganancia de peso $8,68 \mathrm{~g} \mathrm{animal}^{-1}$ $\mathrm{d}^{-1}$. Jiménez et al. (2000) determinaron el momento óptimo económico de beneficio de cuyes alimentados con alfalfa vs una suplementación con afrechillo ambos suministrado ad libitum obteniendo una ganancia de peso 8,60 y 9,40 g animal ${ }^{-1} \mathrm{~d}^{-1}$.

De igual forma, Savón et al. (2006) al utilizar follaje de $M$. alba y concentrado lograron incrementos de peso de 9,30 y $9,70 \mathrm{~g}$ animal ${ }^{-1} \mathrm{~d}^{-1}$, respectivamente. Zeballos et al. (1994) valoraron la hoja de M. alba + concentrado obteniendo valores de 9,70 $\mathrm{g}_{\text {animal }}{ }^{-1} \mathrm{~d}^{-1}$. Sin embargo, estos resultados son inferiores a los reportados por Ramos et al. (2013) cuando utilizaron la harina de nabo ( $B$. campestris) en alimentación de cuyes, obteniendo una ganancia de peso de $13,03 \mathrm{~g}_{\text {animal }}{ }^{-1} \mathrm{~d}^{-1}$. Camino y Hidalgo (2014) analizaron dos genotipos de cuyes alimentados con concentrado y pasto saboya ad libitum, reportando ganancias de 15,60 $\mathrm{g}_{\text {animal }}{ }^{-1} \mathrm{~d}^{-1}$. Sánchez et al. (2013) en el estudio de la respuesta productiva y económica, usando cuatro tipos de comederos para forraje en la crianza de cuyes, la malla colgante en forma de $\mathrm{U}$ reporta valores de $11,10 \mathrm{~g}_{\text {animal }}{ }^{-1} \mathrm{~d}^{-1}$. Apráez et al. (2018) utilizaron M. alba y obtuvieron valores de $11,98 \mathrm{~g}_{\text {animal }}{ }^{-1} \mathrm{~d}^{-1}$. Asimismo, Savón et al. (2006) suministraron M. alba a los cuyes y obtuvieron ganancias entre 10 y $15 \mathrm{~g}_{\text {animal }}{ }^{-1}$ $\mathrm{d}^{-1}$, pero en la evaluación del uso del follaje de $M$. alba y E. poeppigiana obtuvieron 12,27 y $12,15 \mathrm{~g} \mathrm{animal}^{-1}$ $\mathrm{d}^{-1}$, respectivamente. Albert et al. (2006) estudiaron la $M$. alba y la E. poeppigiana en la alimentación de cuyes, reportaron valores de 11,83 y $11,74 \mathrm{~g}_{\text {animal }}{ }^{-1} \mathrm{~d}^{-1}$, respectivamente. Forte y Fernández (1994) utilizaron M. alba en la alimentación de cuyes, reportaron valores de 12,$70 ; 12,40 ; 12,40 \mathrm{~g}_{\text {animal }}{ }^{-1} \mathrm{~d}^{-1}$, respectivamente. Fernández (2002) al utilizar concentrado $+M$. alba fresca obtuvo incrementos de peso de 11,98 $\mathrm{g}$ animal ${ }^{-1} \mathrm{~d}^{-1}$.

El índice de conversión alimenticia no registro diferencia significativa (Cuadro 1) entre las medias de los tratamientos $(\mathrm{P}>0,05)$. Tiene estrecha relación con el consumo de alimento balanceado y la ganancia de peso, Sin embargo (Ramos et al. 2013) asegura que los cuyes, en su condición de animales herbívoros, pueden digerir elementos constituyentes fibrosos de los forrajes, pero su eficiencia es menor que los rumiantes, debido al proceso de fermentación que ocurre en el ciego, por ende, disminuye la ganancia de peso y la conversión alimenticia. Estos resultados son menores a los reportados por Espinel (1999) cuando valora el potencial de uso de árboles y arbustos tropicales y subproductos agrícolas, logrando conversiones alimenticias para la $T$. diversifolia e Hibiscus de 4,95 y 6,78 respectivamente. Apráez et al. (2008) al evaluar el efecto de la M. alba encontraron una conversión alimenticia de 4,78. Forte y Fernández (1994) al utilizar la M. alba y E. poeppigiana obtuvieron valores de 4,56 y 4,56 respectivamente. De la misma forma, Zeballos et al. (1994) reporta valores de 5,50 y 4,10 al utilizar la $M$. alba y morera + concentrado. Estos resultados son más eficientes a lo señalado por Apráez et al. (1997) y Apráez et al. (2001) quienes reportan 8,20 con pasto kikuyo orgánico $50 \%$ / mineral $50 \%+30 \mathrm{~g}$ de concentrado.

De igual forma, la conversión alimenticia $(8,61)$ para el pasto Lolium sp. 70\% y forraje de Abutilon striatum 30\% (Ramos et al., 2013). Apráez et al. (2013) reportan 9,41 de conversión alimenticia utilizando A. scoparius + concentrado comercial. Sánchez et al. (2013) obtuvieron 7,10 de conversión alimenticia al utilizar malla colgante en forma de U. Reportes de conversión alimenticia $(8,04)$ para el forraje + cebada grano + semilla de girasol fueron publicados por Lozada et al. (2013). Albert et al. (2006) encontraron valores para la $M$. alba y E. poeppigiana de 6,87 y 6,87 respectivamente. Liza y Lozano (1994) obtuvieron 6,$87 ; 7,29$ y 8,55 al utilizar ración testigo, 15 y $30 \%$ afrecho de algarroba, respectivamente. Muñoz y Paredes (1994) registraron valores de 7,99 para conversión alimenticia a utilizar E. poeppigiana ad libitum suplementados con $32,10 \mathrm{~g}$ de yuca fresca y 9,90 g concentrado comercial. Apráez et al. (2001) trabajaron con pasto kikuyo orgánico $50 \%$ / mineral 50\% $+30 \mathrm{~g}$ de concentrado, obteniendo 8,20 en la conversión alimenticia. Ramos et al. (2013) evaluaron el pasto Lolium sp. y forraje de A. striatum, logrando valores de 8,61 en la conversión alimenticia.

Por consiguiente, los resultados de la conversión alimenticia se asemejan a los reportados por Quintana et al. (2013) con valores de 5,10 al utilizar alfalfa verde ad libitum + harina de cebada + suplemento mineral. Mientras, Ramos et al. (2013) obtuvieron valores de 5,6 al utilizar pasto Lolium sp. + suplemento comercial. Castillo et al. (2012) reportaron 5,50 para conversión alimenticia al utilizar forraje de maíz chala y suplementación con bloques mineral.

El rendimiento a la canal de cuyes alimentados con balanceado y arbustivas forrajeras tropicales en zona rural de Quevedo, tiene directa relación entre el consumo de alimento, ganancia de peso y conversión alimenticia. Los mejores rendimientos a la canal $(\mathrm{P} \leq 0,01)$ fueron registrados los tratamientos $M$. alba, E. poeppigiana y testigo $(71,93 ; 66,14$ y $64,28 \%)$. Resultados que son superiores a los publicados por Forte y Fernández (1994) 
Cuadro 2. Análisis marginales de los ingresos en cuyes de engorde alimentados con arbustivas forrajeras tropicales

\begin{tabular}{lcccc}
\hline Concepto & $\begin{array}{c}\text { Balanceado } \\
\text { ad libitum }\end{array}$ & $\begin{array}{c}\text { M. alba } \\
\text { ad libitum }\end{array}$ & $\begin{array}{c}\text { E. poeppigiana } \\
\text { ad libitum }\end{array}$ & $\begin{array}{c}\text { T. diversifolia } \\
\text { ad libitum }\end{array}$ \\
\hline Costo $(\mathrm{kg})$ carne $(\$)$ & 9,00 & 9,00 & 9,00 & 9,00 \\
Carne producida $(\mathrm{kg})$ & 0,66 & 0,66 & 0,53 & 0,40 \\
Ingreso venta (\$) & $\mathbf{5 , 9 3}$ & $\mathbf{5 , 9 3}$ & $\mathbf{4 , 7 7}$ & $\mathbf{3 , 6 2}$ \\
\hline
\end{tabular}

cuando evaluaron $30 \mathrm{~g}$ concentrado $+50 \mathrm{~g}$ forraje $M$. alba; $20 \mathrm{~g}$ concentrado $+100 \mathrm{~g}$ forraje M. alba y 15 $\mathrm{g}$ concentrado $+150 \mathrm{~g}$ forraje $M$. alba, obteniendo valores de 61,$00 ; 60,80$ y $59,00 \%$ de rendimiento a la canal, respectivamente. Asimismo, Apráez et al. (2008) realizaron tabajos con $M$. alba y registraron valores de $65,20 \%$. Albert et al. (2006) obtuvieron 65,66 y $65,30 \%$ para la M. alba y E. poeppigiana . Liza y Lozano, (1994) al evaluar los niveles crecientes de 15 y $30 \%$ de afrecho de algarroba y una ración testigo, reportaron valores de $63,97 \% ; 61,18 \%$ y $66,54 \%$ respectivamente. Los valores del rendimiento a la canal se aproximan a los informados por Ramos et al. (2013) con 68,58\%. Mientras que, Ramos et al. (2013) obtuvieron valores de $67,80 \%$ para rendimiento a la canal al utilizar pasto Lolium sp. + suplemento comercial. Sin embargo, estos resultados son inferiores a Camino e Hidalgo (2014) al evaluar dos genotipos de cuyes alimentados con concentrado y exclusión de forraje verde, concentrado+forraje verde y agua reportando un rendimiento a la canal de $73 \%$.

Los mayores ingresos de venta por el peso de carne producida por animal fueron registrados por los tratamientos testigo y M. alba con valores de $\$ 5,93$ cuy $^{-1}$ (Cuadro 2). Los menores costos fijos, costos variables y los costos totales los obtuvo el tratamiento T. diversifolia con $\$ 3,57$ cuy $^{-1}$ (Cuadro 3). El tratamiento M. alba obtuvo el mayor beneficio/costo con $\$ 1,64$ cuy $^{-1}$ en el comportamiento productivo (Cuadro 4). el punto de equilibrio fue $\$ 3,44$ animal $^{-1}$ lo cual demuestra que es una buena alternativa para la crianza de cuyes (Figura 1).

Cuadro 3. Promedios de costos fijos, costos variables y costos totales en cuyes de engorde alimentados con balanceados $\mathrm{y}$ arbustivas forrajeras tropicales

\begin{tabular}{lcccc}
\hline Concepto & $\begin{array}{c}\text { Balanceado } \\
\text { ad libitum }\end{array}$ & $\begin{array}{c}\text { M. alba } \\
\text { ad libitum }\end{array}$ & $\begin{array}{c}\text { E. poeppigiana } \\
\text { ad libitum }\end{array}$ & $\begin{array}{c}\text { T. diversifolia } \\
\text { ad libitum }\end{array}$ \\
\hline Cuyes & 3 & 3 & 3 & 3 \\
Galpón, comederos, bebederos y jaulas & 0,08 & 0,08 & 0,08 & 0,08 \\
Sanidad & 0,02 & 0,02 & 0,02 & 0,02 \\
Total costos fijos & $\mathbf{3 , 1 0}$ & $\mathbf{3 , 1 0}$ & $\mathbf{3 , 1 0}$ & $\mathbf{3 , 1 0}$ \\
Precio (kg) balanceado & 1,62 & 0,40 & 0,40 & 0,40 \\
Precio (kg) forraje & & 0,11 & 0,10 & 0,07 \\
Total costos variables & $\mathbf{1 , 6 2}$ & $\mathbf{0 , 5 1}$ & $\mathbf{0 , 5 0}$ & $\mathbf{0 , 4 7}$ \\
Total Costos & $\mathbf{4 , 7 2}$ & $\mathbf{3 , 6 1}$ & $\mathbf{3 , 6 0}$ & $\mathbf{3 , 5 7}$ \\
\hline
\end{tabular}

Cuadro 4. Rentabilidad en base a promedios de ingresos, egresos y la relación beneficio/costo en cuyes de engorde alimentados con balanceados y arbustivas forrajeras tropicales

\begin{tabular}{lcccc}
\hline Concepto & $\begin{array}{c}\text { Balanceado } \\
\text { ad libitum }\end{array}$ & $\begin{array}{c}\text { M. alba } \\
\text { ad libitum }\end{array}$ & $\begin{array}{c}\text { E. poeppigiana } \\
\text { ad libitum }\end{array}$ & $\begin{array}{c}\text { T. diversifolia } \\
\text { ad libitum }\end{array}$ \\
\hline Ingresos & 5,92 & 5,93 & 4,77 & 3,62 \\
Egresos & 4,72 & 3,61 & 3,60 & 3,57 \\
Beneficio/Costo & $\mathbf{1 , 2 5}$ & $\mathbf{1 , 6 4}$ & $\mathbf{1 , 3 2}$ & $\mathbf{1 , 0 2}$ \\
\hline
\end{tabular}




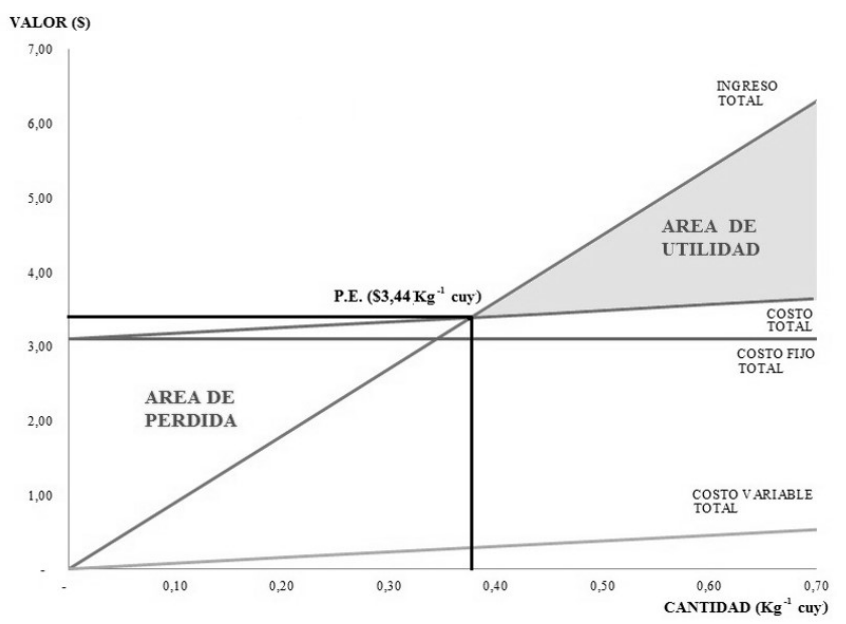

Figura 1. Punto de equilibrio en el comportamiento productivo en cuyes de engorde alimentados con balanceados y arbustivas forrajeras tropicales

\section{Conclusiones}

L os cuyes que consiguieron el mayor consumo de forraje, consumo de alimento, ganancia de peso, rendimiento a la canal y rentabilidad del proceso productivo, fueron los tratamientos a base de balanceado $15 \mathrm{~g}+M$. alba ad libitum y balanceado $15 \mathrm{~g}+$ E. poeppigiana ad libitum). Al suministrar balanceado $15 \mathrm{~g}+T$. diversifolia se afectan los principales parámetros productivos en el engorde de cuyes. El mayor ingreso de venta por carne producida y el mayor beneficio/costo lo registro el tratamiento con balanceado $15 \mathrm{~g}+$ M. alba ad libitum). En términos físicos el punto de equilibrio fue de $\$ 3,44$ animal $^{-1}$, lo cual demuestra que es una buena alternativa para la crianza de cuyes.

\section{Referencia bibliográfica}

Alata, P., Bober, A., Maxime, K. 2008. Ganancia de peso del cuy (Cavia cobayo) con cinco forrajes nativos de Chalhuanca. En: L. Chauca., (ed). Tomo II. Investigaciones en cuyes. Instituto Nacional de Investigaciones Agrarias (INIA), Dirección Nacional de Investigación Agraria. Trabajos presentados en las Reuniones de la Asociación Peruana de producción Animal. Lima, Perú. Pp. 46.

Albert, A., Cruz, M., Rodríguez, S., Savón, L. 2006. Morus alba (morera), Trichantera gigantea (nacedero) y Erythrina poeppigiana (piñón), una opción para la alimentación del Cavia porcellus (cuy). In: V congreso Latinoamericano de Agro-forestaría para la Producción Pecuaria Sostenible. Varadero, Cuba.

Apráez, J., Escobar, E., López, A. 2001. Efecto de la labranza y fertilización orgánica y/o mineral de una pradera de kikuyo (Pennisetum clandestinum) sobre la productividad de cuyes (Cavia porcellus). Revista de
Ciencias Agrícolas. 18(2): 39-49.

Apráez, J., Fernández, L., Hernández, A. 2008. Efecto del empleo de forrajes y alimento no convencionales sobre el comportamiento productivo, rendimiento en canal y calidad de la carne de cuyes (Cavia porcellus). Revista Científica Vet. Zootec. 2(2): 29-34.

Apráez, J., Gómez, T., Calpa, J. 2013. Comportamiento productivo de cuyes (Cavia porcellus) bajo arreglos silvopastoriles en clima medio del departamento de Nariño, Colombia. Revista Investigación Pecuaria. REVIP. 2(2): 41-48.

Apráez, J., Martínez, M., Paredes, F. 1997. Evaluación de dos leguminosas nativas zarza (Mimosa albida) y pepa pega (Desmodium tortuosum) en la alimentación de cuyes (Cavia porcellus). Revista de Ciencias Agrícolas 15 (1:2): 39-49.

Cáceres, O., González, E., Delgado, R. 1996. Valor nutritivo de follaje de árboles y arbustos tropicales. Pastos y Forrajes. 19(1): 27-32.

Camino, J., Hidalgo, V. 2014. Evaluación de dos genotipos de cuyes (Cavia porcellus) alimentados con concentrado y exclusión de forraje verde. Rev Inv Vet Perú. 25(2): 190-197.

Castillo, C., Carcelén, F., Quevedo, W., Ara, M. 2012. Efecto de la suplementación con bloques minerales sobre la productividad de cuyes alimentados con forraje. Rev Inv Vet Perú. 23(4): 414-419.

Espinel, R. 1999. Potencial de uso de árboles y arbustos tropicales y subproductos agrícolas como alimentos para cuyes y conejos. In: V Encuentro Regional sobre Nutrición y producción de Animales Mono gástricos. Maracay, Venezuela. Resumen. Centro para la Investigación en sistemas Sostenibles de Producción Agropecuaria (CIPAV), Cali, Colombia. Pp. 1-12. 
Fernández, L. 2002. Cría y explotación del Cuy (Cavia porcellus) para la producción de carne destinada al consumo humano. [Tesis de doctorado]. [La Habana, Cuba]: Facultad de Agropecuaria de Montaña Escambray (FAME)-Universidad Central de las Villas. Pp. 1-104.

Forte, C., Fernández, C. 1994. Utilización de la morera (Morus alba) en la alimentación de cuyes en crecimiento. In: V Curso y Congreso Latinoamericano de Cuyicultura y mesa redonda sobre Cuyicultura Periurbana. Puerto Ayacucho, Venezuela. Memorias. Centro Nacional para la Producción de Animales de Laboratorio (CENPALAB). Sociedad Nacional de Criadores de Cuyes de Cuba. Pp. 136-141.

Jiménez, A., Bojórquez, C., San Martín, F., Carcelén, F., Pérez, F. 2000. Determinación del momento óptimo económico de beneficio de cuyes alimentados con alfalfa vs. una suplementación con afrechillo. Rev Inv Vet Perú. 11(1): 45-51.

Liza, Z., Lozano, E. 1994. Niveles crecientes de afrecho de algarroba en el crecimiento - acabado de cuyes. En. L. Chauca (ed). Tomo II. Investigaciones en cuyes. Instituto Nacional de Investigaciones Agrarias (INIA), Dirección Nacional de Investigación Agraria. Trabajos presentados en las Reuniones de la Asociación Peruana de producción Animal. Lima, Perú. Pp. 41.

Lozada, P., Jiménez, R., San Martín, F., Huamán, A. 2013. Efecto de la inclusión de cebada grano y semilla de girasol en una dieta basada en forraje sobre el momento óptimo de beneficio de cuyes. Rev Inv Vet Perú. 24 (1):25-31.

Meza, G., Cabrera, R., Morán, J., Meza, F., Cabrera, C., Meza, C., Meza, J., Cabanilla, M., López, F., Pincay, J., Bohórquez, T., Ortiz, J. 2014. Mejora de engorde de cuyes (Cavia porcellus L.) a base de gramíneas y forrajeras arbustivas tropicales en la zona de Quevedo, Ecuador. IDESIA. 32(3): 75-80.

Muñoz, M., Paredes, M. 1994. Alimentación de cuyes con eritrina (Erythrina sp.), suplementada con yuca fresca (Manihot esculenta) y concentrado comercial. En. L. Chauca (ed). Tomo II. Investigaciones en cuyes. Instituto Nacional de Investigaciones Agrarias (INIA), Dirección Nacional de Investigación Agraria. Trabajos presentados en las Reuniones de la Asociación Peruana de producción Animal. Lima, Perú. Pp. 153.
Quintana, E.; Jiménez, R., Carcelén, F., San Martín, F., Ara, M. 2013. Efecto de dietas de alfalfa verde, harina de cebada y bloque mineral sobre la eficiencia productiva de cuyes. Rev Inv Vet Perú. 24(4): 425-432.

Ramos, L., Chamorro, M., Benavides, J. 2013. Evaluación de harina de nabo (Brassica campestris) en alimentación de cuyes (Cavia porcellus). Revista Investigación Pecuaria. 2(2): 33-40.

Ramos, L., Guevara, A., Villota, M. 2013. Evaluación del comportamiento productivo de cuyes (Cavia porcellus) alimentados con pasto aubade (Lolium sp.) y forraje de abutillón (Abutilon striatum). Revista Investigación Pecuaria. REVIP. 2(2): 23-31.

Sánchez, A., Sánchez, S., Godoy, S., Díaz, R., Vega, N. 2009. Gramíneas tropicales en el engorde de cuyes mejorados sexados (Cavia porcellus Linnaeus) en la zona de la Mana. Revista Ciencia y Tecnología. Ecuador. 2: 25-28.

Sánchez, A, Zambrano, D, Torres, E, Meza, G. 2012. Forrajeras tropicales y banano maduro (Musa paradisiaca) en el engorde de cuyes (Cavia porcellus L.) en el cantón Quevedo. AICA. (2): 287-290.

Sánchez, R., Jiménez, R., Huamán, H., Bustamante, J., Huamán, A. 2013. Respuesta productiva y económica al uso de cuatro tipos de comederos para forraje en la crianza de cuyes. Rev Inv Vet Perú. 24(4): 441-450.

Savón, L., Ly, L., Albert, A., Dihigo, L. 2006. Avances en el uso del follaje de morera en la alimentación de especies monogástrico. In: IV Congreso Latinoamericano de Agro-forestaría para la Producción Pecuaria Sostenible. Varadero, Cuba.

SAS, 2000. SAS user's guide: version 8. (SAS Institute: Cary, $\mathrm{NC})$.

Zeballos, L., Hidalgo, V., Moreno, A., Montes, T. 1994. Evaluación biológica de la morera mediante pruebas de digestibilidad y crecimiento en cuyes. En. L. Chauca (ed). Tomo II. Investigaciones en cuyes. Instituto Nacional de Investigaciones Agrarias (INIA), Dirección Nacional de Investigación Agraria. Trabajos presentados en las Reuniones de la Asociación Peruana de Producción Animal. Lima, Perú. Pp. 172. 